\title{
Cotidianidad familiar y comunitaria con relación al desarrollo humano en familias del barrio La Panela del municipio de Corozal, Sucre
}

Blanca Pérez Contreras

\section{Resumen}

Este artículo analiza la cotidianidad familiar y comunitaria en relación con el desarrollo humano, tomando como referente las siguientes dimensiones: composición familiar, condiciones educativas, económicas y habitacionales, hábitat, comunicación familiar, relaciones de vecindad, resolución de conflictos comunitarios y actividades compartidas en la comunidad. Se prioriza una perspectiva integradora mas no individualizada de la cotidianidad familiar y comunitaria, concibiendo estos dos ámbitos como ejes dinamizadores y catalizadores de desarrollo humano en cuanto a espacios de socializacion, proteccion, afecto, cuidado y bienestar. No obstante, los resultados muestran que los miembros adultos presentan baja preparación académica, lo que incide en el tipo de actividad economica y 10 trabajo que realizan, en los ingresos percibidos en la familia y por ende en la forma como satisfacen las necesidades de alimentacion, vestido, vivienda, salud y recreación. En tal sentido, el estudio aporta información y sugiere líneas de acción a instituciones públicas y privadas, a la academia y a profesionales para que formulen programas y estrategias integrales dirigidas a la atencion temprana y la prevención de factores de riesgo que puedan afectar el bienestar biopsicosocial de los integrantes de las familias. El diseño empleado en esta investigación fue no experimental; el tipo de estudio, observacional;

1 Universidad Nacional Abierta y a Distancia, Tutora; Socióloga, Magister en Educación: Sociología de la Educación. Docen te e Investigadora en la Corporación Universitaria del Caribe - Cecar. Experiencia laboral de 16 años en Educación Superio modalidad Presencial y a Distancia; directora del Grupo de Investigación Montes de María, Categoría D-Colciencias, blanca. perez@unad.edu.co. 


\title{
BancaPéezContreres
}

Cotidianidad familiar y comunitaria con relación al desarrollo humano en familias del barrio La Panela del municipio de

Corozal, Sucre, artículo producto de la investigación

el nivel, descriptivo-interpretativo y el método, mixto, integrado con datos cuantitativos y cualitativos según Shoveller (2006).

Palabras clave: Vida cotidiana, familia, comunidad, desarrollo humano, habitabilidad, comunicación familiar.

\section{Family and community daily life in human development among families in La Panela neighborhood in the municipality of Corozal, Sucre}

\begin{abstract}
Abstrack
This article examines family and community daily life and its relationship to human development, having as a reference the following dimensions: family composition, educational, economic and housing conditions, habitat, family communication, relations with neighbors, conflict resolution and community shared activities. Priority is given to an integrating rather than individualized perspectivo of family and community everyday life, conceiving these two areas as energizing lines and catalysts of human development in terms of social spaces, protection, fondness, care and welfare. However, the results show that adult members have low academic preparation, focusing on the type of economic activity and/or work performed, in family income and therefore how they meet their needs for food, clothing, housing, health and recreation. In this regard, the study provides information and suggests courses of action for private and public institutions, the academy and professionals for the development of comprehensive programs and strategies aimed to early intervention and prevention of risk factors that may affect family members' biopsychosocial welfare. The design used in this research was not experimental, the type of observational study, the descriptive and interpretative level and mixed method, comprising quantitative and qualitative data, according to Shoveller (2006).
\end{abstract}

Key words: Daily life, family, community, human development, housing, family communication.

Recibido: 2 Marzo de 2011

Aceptado: 20 Mayo de 2011 
Revista de

investigaciones UNAD

Volumen 10. Número 1. Junio 2011

\section{Introducción}

Los cambios sociales, culturales económicos y ecológicos que se han dado en Colombia a partir de los años 80 , han incidido en el incremento de la marginalidad urbana y la pobreza, afectando la vida cotidiana de vastos sectores de población y la forma de satisfacer las necesidades de educacion, salud, vivienda, trabajo y ambientes saludables, según Virginia Gutierrez de Pineda (1991). Por ello, el estudio de la cotidianidad familiar en toda su compleilidad, requiere una comprension multidimensional de la manera como transcurre la vida, a partir de la identificacion de aspectos objetivos y subjetivos de su existencia.

La investigación se centró en "la cotidianidad familiar y comunitaria en relacion con el desarrollo humano de las familias del barrio La Panela de municipio de Corozal Sucre" Se trata de un contexto sociocultural donde el ideal de desarrollo de las familias y la comuni dad se ve truncado por la falta de iniciativa, conocimientos y, recursos economicos para trabajar individual y/o colectivamente en la solución de, sus problemas y necesidades;' así mismo, por la inexistencia de una politica publica que contribuya a mejorar la calidad de vida, por una parte, de la poblacion infantil y adolescente, segun lo señalado en los artículos 8 y 29 del Código de la Infancia y A dolescencia (2006) y por la otra, de adul tos y ancianos. L a cali idad de vida, segun Sabeh, E. N. y Verdugo, M. A. (2000), se refiere a la satisfacción percibida por una persona o grupo en diferentes ambitos de su vida.

El abordaje integral que se decidió hacer para el estudio de la cotidianidad familiar y comunitaria llevo a considerar varias de las dimensiones propuestas por estos teoricos, entre ell as la forma como satisfacen sus necesidades sociales y materiales, la inclusion social y las condiciones del ambiente, es decir se prioriza una perspectiva integradora de, lo familiar y comunitario pero no individualizada, concibiendo estos dos ámbitos como ejes dinamizadores y catalizadores de desarrollo humano, Con ello se busca aportar informacion que sirva de base a instituciones publicas y privadas, a la academia y a los profesionales para que formulen programas y estrategias integrales para la atencion temprana y la prevencion de factores de riesgo que puedan afectar el bienestar biopsicosocial de los miembros de la familia. Parafrasęando a M aldonado (1995), se asume la familia como "un grupo social sistemico que se caracteriza por la interacción permanente entre sus miembros, pero al mismo tiempo como el lugar donde se estructura la identidad, se protege a los mi embros y se da el intercambio con la cul tura y lo comunitario".

De este modo, hablar de la cotidianidad familiar y comunitaria en relación con el desarrollo humano alude a las posibilidades de acceso y disfrute de bienes materiales y sociales, al goce de ambiente sano y vivienda de calidad y al acceso a la educacion y al trabajo en condiciones de dignidad. desarrollo humano, segun Delgado de Bravo y Failache (1993), es "el grado 


\section{BancaPérezContreres}

Cotidianidad familiar y comunitaria con relación al desarrollo humano en familias del barrio La Panela del municipio de

Corozal, Sucre, artículo producto de la investigación

de bienestar de las comunidades y sociedades determinado por la satisfacción de sus necesidades fundamentales, en procura de asegurarse su existencia, permanencia y trascendencia en un espacio dado y en un momento historico determinado". Es ese conjunto de satisfactores, situaciones, experiencias y relaciones determinadas por las interacciones diarias, a traves de lo que los antropologos han denominado papeles y/o roles, que para Hannerz (1980), pueden darse en cinco ambitos: domestico y de parentesco, aprovisionamiento, recreacion, vecindad y transito.

En suma, el artículo da cuenta de la cotidianidad familiar y comunitaria y sus afectaciones en el desarrollo humano de los habitantes del barrio La Panela.

\section{Metodología}

Método y tipo de estudio. Respecto al método, en una relación de complementariedad se tomaron elementos de la investigacion cuantitativa y cual itativa segun Campos (2009), buscando entender lo que ocurre en el microsistema familiar y comunitario a traves del reconocimiento que sus miembros hacen de la realidad vivida en el dia a dia, informacion que se apoya con tablas de frecuencia y combinadas. En concordancia con el enfogue, el diseño es no experimental, el tipo de estudio es observacional y el nivel es descriptivo-interpretativo.

Participantes. La unidad de análișis y observación la constituyeron 104 de las 120 familias que viven en el barrio La Panela y por ello no se requirio hacer muestreo, Los criterios de seleccion estuvieron determinados por el interes y motivacion para participar en el estudio.

Instrumentos. Se aplicó una entrevista semiestructurada a un miembro adulto que podia ser padre, madre 0 abuelo. El cuestionario estuvo constituido por 34 preguntas entre ábiertas y cerradas, elaboradas por la investigadora y por participantes del semillero, sometidas a fiabilidad a traves del juicio de expertos.

Procedimiento. La ejecución del proyecto en campo necesitó el apoyo de la presidenta de la junta de Acción Comunal del barrio, quien facilito su socialización a las familias, obteniendo de estas su consentimiento para la recolección de la información. Posteriormente se realizaron las siguientes acciones:

1. Recabar en la ịnformación y en su análisis estadístico-descriptivo, lo que hizo necesario organizarla, agrupando las respuestas obtenidas en cada item de acuerdo con similitudes y diferencias entre los aspectos estudiados (A nguera, 1998). 
Revista de

investigaciones UNAD

Volumen 10. Número 1. Junio 2011

2. Codificar y preparar la matriz de datos, utilizando el programa estadistico SPSS.

3. Tabular y analizar los resultados por categorías y subcategorías, que dieran origen a las conclusiones.

\section{Resultados y discusión}

En la presentación de los resultados se utilizan tablas de frecuencia y combinadas, en las que se puede identificar la variable a analizar, la frecuencia (F) y el porcentaje (\%) de cada opción de respuesta. A tendiendo la perspectiva integral que se priorizo en la investigacion, se presentan a continuacion los aspectos relacionados con la cotidianidad familiar y comunitaria, los ambitos de interaccion y los generadores de desarrollo humano. Veamos:

Tabla 1. Lugar de procedencia de las familias

\begin{tabular}{|c|c|c|}
\hline ZONAS DE ORIGEN & F & $\%$ \\
\hline Rural & 35 & 33,5 \\
\hline Barrios de la cabecera municipal & 63 & 60,6 \\
\hline No responde & 6 & 5,8 \\
\hline Total & 104 & 100 \\
\hline
\end{tabular}

Fuente: Padres de familias participantes en la investigación, 2010

La tabla 1 muestra que el 66\% de las familias son procedentes de la cabecera municipal, por movilidad interbarrial y el $34 \%$ de zonas rurales como consecuencia del conflicto armado.

Tabla 2. Tipo de unión conyugal

\begin{tabular}{|c|c|c|}
\hline VíNCULO CONYUGAL & F & $\%$ \\
\hline Unión libre & 53 & 51 \\
\hline Casado & 32 & 30,8 \\
\hline Separado & 12 & 11,5 \\
\hline Viudo & 3 & 2,9 \\
\hline No responde & 4 & 3,8 \\
\hline Total & 104 & 100 \\
\hline
\end{tabular}

Fuente: Padres de familias participantes en la investigación, 2010 


\section{BancaPéezContreres}

Cotidianidad familiar y comunitaria con relación al desarrollo humano en familias del barrio La Panela del municipio de

Corozal, Sucre, artículo producto de la investigación

Según la tabla 2, el estado civil que prevalece es la unión libre con un 51.5\%; siguen los casados (31.1\%), los separados (10.7\%) y los viudos.

Tabla 3. Distribución de población infantil y adolescentes por edad

\begin{tabular}{|c|c|c|c|c|c|}
\hline INFANCIA & $\mathbf{F}$ & $\%$ & ADOLESCENTES & $\mathbf{F}$ & $\%$ \\
\hline 0 a 2 años & 33 & 31,7 & 12 a 14 años & 48 & 46,2 \\
\hline 3 a 5 años & 34 & 32.7 & 15 a 17 años & 36 & 34,6 \\
\hline 6 a 8 años & 26 & 25 & 18 a 20 años & 15 & 14,4 \\
\hline 9 a 11 años & 11 & 10,6 & 21 a 23 años & 5 & 4,8 \\
\hline Total & 104 & 100 & Total & 104 & 100 \\
\hline
\end{tabular}

Fuente: Padres de familias que participaron en la investigación, 2010

La tabla 3 presenta la distribución de la composición familiar de niños/as y adol escentes de ambos sexos, por grupos de edades. Entre los niños se encontro que la mayor representatividad la tiene la primera infancia con el 89.4\%; el $10.6 \%$ esta en la segunda infancia (9 a 11 año.s). Los adolescentes en un 46.2\% estan en la adol escencia temprana (12 a 14 anos) y el, $53.8 \%$ se halla en edades que oscilan entre los 15 y 23 años (adolescencia tardía).

Tabla 4. Distribución de la población adulta y adulta mayor según rango de edad

\begin{tabular}{|c|c|c|c|c|c|}
\hline ADULTOS/AS & $\mathbf{F}$ & $\%$ & $\begin{array}{c}\text { ADULTO } \\
\text { MAYOR }\end{array}$ & $\mathbf{F}$ & $\%$ \\
\hline 24 a 26 años & 41 & 39,8 & 51 a 55 años & 41 & 39,8 \\
\hline 27 a 29 años & 36 & 35 & 56 a 60 años & 32 & 31,1 \\
\hline 30 a 32 años & 11 & 10,7 & 61 a 65 años & 11 & 10,7 \\
\hline 33 a 35 años & 10 & 9,7 & 66 a 70 años & 7 & 6,8 \\
\hline 36 a 38 años & 2 & 1,9 & 71 a 75 años & 7 & 6,8 \\
\hline 39 a 41 años & 4 & 2.8 & 76 a 86 años & 6 & 4,8 \\
\hline Total & 104 & 100 & Total & 104 & 100 \\
\hline
\end{tabular}

Fuente: Padres de familias participantes en la investigación, 2010

La tabla 4 registra la composición de los adultos y adultos mayores así: el $95.2 \%$ son adultos en edades de 24 a 35 años y el $4.8 \%$ de 36 a 41 años. En los adultos mayores prevalece la edad de 51 a 60 años con un $70.9 \%$; el resto está entre los 61 y 86 años. 
Revista de

investigaciones UNAD

Volumen 10. Número 1. Junio 2011

Tabla 5. Población infantil con registro de nacimiento

\begin{tabular}{|c|c|c|}
\hline CON REGISTRO & F & $\%$ \\
\hline Con registro de 0-8 años & 78 & 75 \\
\hline Sin registro civil & 17 & 16,3 \\
\hline Solo dos de cuatro niños & 9 & 8,7 \\
\hline Total & 104 & 100 \\
\hline
\end{tabular}

Fuente: Padre, madre que participaron en la investigación, 2010

Según los datos de la tabla 5, el 75\% de niños y niñas tienen registro civil, el $16.3 \%$ no cuentan con él y en 9 familias solo 2 de 4 hijos e hijas lo poseían.

Tabla 6. Adolescentes y adultos con documento de identificación

\begin{tabular}{|c|c|c|c|c|c|}
\hline $\begin{array}{c}\text { TARJETA } \\
\text { IDENTIDAD }\end{array}$ & $\mathbf{F}$ & $\%$ & CÉDULA & $\mathbf{F}$ & $\%$ \\
\hline $\begin{array}{c}\text { Entre } 8 \text { y } 18 \\
\text { años }\end{array}$ & 65 & 62,5 & SI & 93 & 89.4 \\
\hline $\begin{array}{c}\text { No tienen } \\
\text { tarjeta }\end{array}$ & 33 & 31,7 & NO & 8 & 7.7 \\
\hline No responde & 6 & 5,8 & No responde & 3 & 2,9 \\
\hline Total & 104 & 100 & Total & 104 & 100 \\
\hline
\end{tabular}

Fuente: Padre, madre que participaron en la investigación, 2010

Según la tabla 6, el 62.5\% posee tarjeta de identidad, el 31.7\% no la acreditan y el resto no respondio. La cedula de ciudadania la tiene el 89.4\%; esta indocumentado el $7,7 \%$, lo que puede acarrearles problemas con la ley e inconvenientes para desplazarse dentro y fuera del municipio e incluso en caso de ingreso laboral formal.

Tabla 7. Nivel de escolaridad de la población adulta

\begin{tabular}{|c|c|c|c|}
\hline NIVEL EDUCATIVO & F & \% & \% ACUMULADO \\
\hline $\begin{array}{c}\text { Básica primaria } \\
\text { incompleta }\end{array}$ & 51 & 49 & 49 \\
\hline $\begin{array}{c}\text { Básica secundaria (6o } \\
\text { y } 7^{\circ} \text { grado) }\end{array}$ & 23 & 22,1 & 71,1 \\
\hline Técnico sin título & 18 & 17,4 & 88,5 \\
\hline
\end{tabular}




\section{BancapéezContreres}

Cotidianidad familiar y comunitaria con relación al desarrollo humano en familias del barrio La Panela del municipio de

Corozal, Sucre, artículo producto de la investigación

\begin{tabular}{|c|c|c|c|}
\hline NIVEL EDUCATIVO & F & \% & \% ACUMULADO \\
\hline Sin escolaridad & 12 & 11,5 & 100 \\
\hline Total & 104 & 100 & \\
\hline
\end{tabular}

Fuente: Datos obtenidos de los adultos entrevistados, 2010

Los datos de la tabla 7 señalan que el nivel educativo del 71,1\% de los padres y otros miembros adultos oscila entre primaria y secundaria incompleta; el $17.4 \%$ han realizado cursos en oficios técnicos y el $11.5 \%$ son iletrados.

Tabla 8. Escolaridad de la población infantil y adolescente

\begin{tabular}{|c|c|c|c|}
\hline Nivel educativo & $\mathbf{f}$ & $\%$ & \% Acumulado \\
\hline Preescolar & 21 & 20,2 & 20,2 \\
\hline Primaria completa & 65 & 62,5 & 82,7 \\
\hline Secundaria incompleta & 12 & 11,5 & 94,2 \\
\hline No estudian & 6 & 5,8 & 100 \\
\hline Total & 104 & 100 & \\
\hline
\end{tabular}

Fuente: Padre o madre de familia entrevistado/a, 2010

Según la tabla 8, la escolaridad oscila entre primaria completa (62.5\%), secundaria incompleta $(11.5 \%)$, preescolar $20.2 \%$ y sin estudio $5.8 \%$.

Tabla 9. Niños y niñas que requieren educación especial

\begin{tabular}{|c|c|c|c|}
\hline $\begin{array}{c}\text { EDUCACIÓN } \\
\text { ESPECIAL }\end{array}$ & $\mathbf{F}$ & \% & \% ACUMULADO \\
\hline SI & 4 & 3,8 & 3,8 \\
\hline NO & 100 & 96,2 & 100 \\
\hline Total & 104 & 100 & \\
\hline
\end{tabular}

Fuente: Informantes padres de familias entrevistados, 2010

La tabla 9 referencia un $3.8 \%$ de los niños y niñas con necesidades de educacion especial, los cuales no son atendidos en la institucion que funciona en el municipio. 
Revista de

investigaciones UNAD

Volumen 10. Número 1. Junio 2011

Tabla 10. Personas que trabajan y tipo de ocupación de los adultos

\begin{tabular}{|c|c|c|c|c|c|}
\hline $\begin{array}{c}\text { PROVEEDOR } \\
\text { ECONÓMICO }\end{array}$ & $\mathbf{F}$ & $\%$ & $\begin{array}{c}\text { TIPO DE } \\
\text { OCUPACIÓN }\end{array}$ & $\mathbf{F}$ & $\%$ \\
\hline Ambos padres & 32 & 30,8 & $\begin{array}{c}\text { Trabajo } \\
\text { independiente }\end{array}$ & 45 & 43,3 \\
\hline Solo la madre & 22 & 21,2 & Obrero & 31 & 29,8 \\
\hline $\begin{array}{c}\text { Solo el padre } \\
\text { Hermanos } \\
\text { mayores }\end{array}$ & 35 & 33,7 & $\begin{array}{c}\text { Empleada } \\
\text { doméstica }\end{array}$ & 9 & 8,7 \\
\hline Padres e hijos & 8 & 6,7 & Jornalero & 11 & 10,6 \\
\hline Total & 104 & 100 & Total & 104 & 100 \\
\hline
\end{tabular}

Fuente: Padres de familias entrevistados, 2010

Respecto a las personas que trabajan (tabla 10), el padre tiene una participación del $33.7 \%$; ambos padres el 30.8\%, la madre el 21,2\% y los hijos mayores el 6.7\%. En cuanto al tipo de ocupacion, el trabaio independiente tiene el $43.3 \%$, obrero y jornalero el 40.4\%, domésticas y trabajadores en sus parcelas el 16.3\%.

Tabla 11. Duración de la jornada de trabajo y forma de pago

\begin{tabular}{|c|c|c|c|c|c|}
\hline DURACIÓN & $\mathbf{F}$ & $\%$ & INGRESO & $\mathbf{F}$ & $\%$ \\
\hline 3 a 4 horas & 17 & 16,3 & Diario & 67 & 64,4 \\
\hline 5 a 6 horas & 27 & 26 & Semanal & 17 & 16,3 \\
\hline 7 a 8 horas & 27 & 26 & Quincenal & 4 & 3,9 \\
\hline 9 a 10 horas & 33 & 31,7 & Mensual & 16 & 15,4 \\
\hline Total & 104 & 100 & Total & 104 & 100 \\
\hline
\end{tabular}

Fuente: Personas adultas que trabajan en el hogar, 2010

La tabla 11 registra una jornada de trabajo que oscila entre 3 y 10 horas. El ingreso recibido es diario en $64.4 \%$, semanal en $16.3 \%$, quincenal en $3.8 \%$ y mensulal en $15.4 \%$.

Tabla 12. Tipo de habitación y material de construcción de las viviendas

\begin{tabular}{|c|c|c|}
\hline TENENCIA & F & $\%$ \\
\hline Propia y la están pagando & 20 & 19,2 \\
\hline Propia, totalmente pagada & 52 & 50.1 \\
\hline Arrendada & 17 & 16,3 \\
\hline
\end{tabular}




\section{BancapéezContreres}

Cotidianidad familiar y comunitaria con relación al desarrollo humano en familias del barrio La Panela del municipio de

Corozal, Sucre, artículo producto de la investigación

\begin{tabular}{|c|c|c|}
\hline TENENCIA & F & \% \\
\hline Familiar & 15 & 14,4 \\
\hline Total & 104 & 51 \\
\hline Material de construcción & F & \\
\hline $\begin{array}{c}\text { Paredes de concreto y techo } \\
\text { de Eternit }\end{array}$ & 53 & 12,5 \\
\hline $\begin{array}{c}\text { Paredes de bahareque y techo } \\
\text { de palma }\end{array}$ & 13 & 35,6 \\
\hline Concreto y techo de zinc & 37 & 0.9 \\
\hline No responde & 1 & 100 \\
\hline Total & 104 & \\
\hline
\end{tabular}

Fuente: Información suministrada por los padres de familia entrevistados, 2010

La tabla 12 muestra que el $50.1 \%$ vive en casa propias y libres de hipotecas, el 19. $2 \%$ la estan pagando y el resto son arrendadas o familiares. Los materiales de construccion que predominan son concreto y Eternit y concreto y zinc, en tanto que la pal ma y bahareque son materiales de calidad bastante baja.

Tabla 13. Distribución espacial de la vivienda y personas que duermen por cuarto

\begin{tabular}{|c|c|c|c|c|c|}
\hline HABITACIONES & F & $\%$ & $\begin{array}{c}\text { PERSONAS X } \\
\text { CUARTO }\end{array}$ & F & $\%$ \\
\hline Un cuarto & 45 & 43,3 & 3 a 4 personas & 32 & 30,8 \\
\hline Dos cuartos & 47 & 45,2 & 5 a 6 personas & 46 & 44,2 \\
\hline Tres cuartos & 12 & 11,5 & 7 a 8 personas & 26 & 25 \\
\hline Total & 104 & 100 & Total & 104 & 100 \\
\hline
\end{tabular}

Fuente: Padres de familia entrevistados, 2010

La tabla 13 referencia el $43.3 \%$ de viviendas con un cuarto, el $45.2 \%$ con dos y el $11.5 \%$ con tres. Significa que el $56.7 \%$ de las viviendas por el numero de integrantes de la familia (de 3 a 4 o de 5 a 8 personas) no cumplen con las condiciones de viviendas dignas. Solo el $11.5 \%$ cuenta con cocina independiente y el resto prepara los a imentos en ramadas improvisadas y construidas en el patio.

Tabla 14. Servicios públicos con que cuenta la vivienda y calidad de los mismos

\begin{tabular}{|c|c|c|c|c|c|}
\hline SERVICIOS QUE POSEEN & F & $\%$ & CALIDAD & F & $\%$ \\
\hline Agua, luz y alcantarillado & 36 & 34,6 & Bueno & 29 & 27,9 \\
\hline
\end{tabular}

90 
Revista de

investigaciones UNAD

Volumen 10. Número 1. Junio 2011

\begin{tabular}{|c|c|c|c|c|c|}
\hline SERVICIOS QUE POSEEN & F & $\%$ & CALIDAD & F & $\%$ \\
\hline Agua, gas y luz & 22 & 21,2 & Regular & 57 & 54,8 \\
\hline Agua y luz & 46 & 44,2 & Malo & 18 & 17,3 \\
\hline Total & 104 & 100 & Total & 104 & 100 \\
\hline
\end{tabular}

Fuente: Padres de familia entrevistados, 2010

Según la tabla 14, los servicios de agua y luz tienen un cubrimiento del 100\%; fueron calificados entre reqular y malo por el $72.1 \%$ de las familias. El al cantarillado beneficia solo al 34.6\%; la falta del mismo es suplida por un $44.2 \%$ de las familias con inodoros conectados a pozo septico, y otras personas hacen sus necesidades y las tiran a cielo abierto. El servicio de gas, no lo tiene el $78.8 \%$ de las familias, y a cambio, utilizan hornillas, fogon de leña y gasolina.

Tabla 15. Características de la comunicación en el núcleo familiar

\begin{tabular}{|c|c|c|}
\hline CARACTERISTICAS & F & $\%$ \\
\hline Diálogo y mutuo entendimiento & 51 & 20,2 \\
\hline $\begin{array}{c}\text { Discordias entre padres y entre } \\
\text { padres e hijos }\end{array}$ & 21 & 14,4 \\
\hline $\begin{array}{c}\text { Conflicto entre hermanos y } \\
\text { diálogo con padres }\end{array}$ & 15 & 8,7 \\
\hline $\begin{array}{c}\text { A veces desacuerdos/entendi- } \\
\text { miento entre hermanos y entre } \\
\text { padres }\end{array}$ & 5 & 4,8 \\
\hline $\begin{array}{c}\text { Desacuerdos entre hermanos y } \\
\text { otros familiares }\end{array}$ & 3 & 2,9 \\
\hline No responde & 104 & 100 \\
\hline Total & 9 & \\
\hline
\end{tabular}

Fuente: Padres de familia entrevistados, 2010

Según la tabla anterior, el 49\% utiliza el diálogo y el mutuo entendimiento; el $34.6 \%$ dijo que existen discordias entre padres, entre padres e hijos y entre hermanos y otros familiares; la dualidad diálogo-desacuerdo entre hermanos y entre padres tiene un $8.7 \%$. 


\section{BancapéezContreres}

Cotidianidad familiar y comunitaria con relación al desarrollo humano en familias del barrio La Panela del municipio de Corozal, Sucre, artículo producto de la investigación

Tabla 16. Reglas y normas que regulan la convivencia familiar

\begin{tabular}{|c|c|c|}
\hline REGLAS & F & $\%$ \\
\hline Respeto y tolerancia & 27 & 26 \\
\hline $\begin{array}{c}\text { Horas de acostarse y realiza- } \\
\text { ción de tareas }\end{array}$ & 18 & 17,3 \\
\hline Responsabilidad & 11 & 10,6 \\
\hline Ayuda mutua y cooperación & 20 & 19,2 \\
\hline $\begin{array}{c}\text { Horario de salida y llegada a } \\
\text { casa }\end{array}$ & 10 & 9,6 \\
\hline Respeto a los mayores & 12 & 11,5 \\
\hline No sabe & 6 & 5,8 \\
\hline Total & 104 & 100 \\
\hline
\end{tabular}

Fuente: Padres de familia entrevistados, 2010

En general, el $94.2 \%$ de las familias pone en práctica las normas de convivencia y solo el $5.8 \%$ dijo no saber que las reglas y las normas son importantes en la familia.

Tabla 17. Persona(s) que imponen normas en la familia

\begin{tabular}{|c|c|c|}
\hline IMPONEN NORMAS & F & $\%$ \\
\hline Mamá & 27 & 25.9 \\
\hline Papá & 23 & 22.1 \\
\hline Ambos padres & 46 & 44.2 \\
\hline Abuelos y hermanos mayores & 8 & 7.8 \\
\hline Total & 104 & 100 \\
\hline
\end{tabular}

Fuente: Padres, abuelos participantes en la investigación, 2010

La imposición de normas está a cargo de ambos padres en el 44.2\%; la madre lo hace el $25.9 \%$; el padre, el $22.1 \%$ y los abuelos y hermanos mayores, el $7.8 \%$.

Tabla 18. Horas compartidas entre los miembros de la familia

\begin{tabular}{|c|c|c|}
\hline HORAS & F & $\%$ \\
\hline Horas de la mañana & 34 & 32,7 \\
\hline Horas de la tarde & 11 & 10,6 \\
\hline
\end{tabular}

92 
Revista de

investigaciones UNAD

Volumen 10. Número 1. Junio 2011

\begin{tabular}{|c|c|c|}
\hline HORAS & F & $\%$ \\
\hline Todo el día & 10 & 9,5 \\
\hline Horas de la noche & 22 & 21,2 \\
\hline Nunca & 27 & 26 \\
\hline Total & 104 & 100 \\
\hline
\end{tabular}

Fuente: Padres entrevistados, 2010

El 74\% de las familias respondieron que comparten al gunas horas en la mañana, otros en la noche o en la tarde (observese la discriminacion en la tabla 18), mientras que el $26 \%$ nunca lo hace. Las obligaciones fuera del hogar, inciden en la cantidad y calidad del tiempo compartido.

Tabla 19. Organización del uso del tiempo en los miembros de la familia

\begin{tabular}{|c|c|c|c|c|c|}
\hline COMIDA & $\mathbf{F}$ & $\%$ & TAREAS & $\mathbf{F}$ & $\%$ \\
\hline SI & 68 & 65,4 & SI & 86 & 82,7 \\
\hline NO & 36 & 34,6 & NO & 18 & 17,3 \\
\hline Total & 104 & 100 & Total & 104 & 100 \\
\hline Asearse & $\mathrm{F}$ & $\%$ & $\begin{array}{c}\text { Juego y ver } \\
\text { TV }\end{array}$ & $\mathrm{F}$ & $\%$ \\
\hline SI & 53 & 51 & SI & 62 & 59,6 \\
\hline NO & 51 & 49 & NO & 42 & 40,4 \\
\hline Total & 100 & 100 & Total & 104 & 100 \\
\hline
\end{tabular}

Fuente: Padres de familia entrevistados, 2010

El uso de horarios en las actividades familiares se establece para la comida en el $65.4 \%$, para hacer tareas en el $82.7 \%$, para asearse en el $51 \%$ y para jugar y ver television en el $62 \%$. El porcentaje de familias que no se sujetan a horarios oscila entre $17.3 \%$ y $49 \%$.

Tabla 20. Presencia de factores contaminantes en el barrio La Panela

\begin{tabular}{|c|c|c|c|c|c|}
\hline $\begin{array}{c}\text { AGUAS } \\
\text { EMPOZADAS }\end{array}$ & $\mathbf{F}$ & $\%$ & $\begin{array}{c}\text { LOTES } \\
\text { ENMONTADOS }\end{array}$ & $\mathbf{F}$ & $\%$ \\
\hline SI & 34 & 32,7 & SI & 55 & 52,9 \\
\hline NO & 70 & 67,3 & NO & 49 & 47,1 \\
\hline Total & 104 & 100 & Total & 104 & 100 \\
\hline
\end{tabular}




\section{BancapéezContreres}

Cotidianidad familiar y comunitaria con relación al desarrollo humano en familias del barrio La Panela del municipio de Corozal, Sucre, artículo producto de la investigación

\begin{tabular}{|c|c|c|c|c|c|}
\hline $\begin{array}{c}\text { AGUAS } \\
\text { EMPOZADAS }\end{array}$ & $\mathbf{F}$ & $\%$ & $\begin{array}{c}\text { LOTES } \\
\text { ENMONTADOS }\end{array}$ & $\mathbf{F}$ & $\%$ \\
\hline Presencia Basuras & $\mathrm{F}$ & $\%$ & Calles destapadas & $\mathrm{F}$ & $\%$ \\
\hline SI & 20 & 19,2 & $\mathrm{SI}$ & 104 & 100 \\
\hline NO & 84 & 80.8 & NO & - & - \\
\hline Total & 104 & 100 & Total & 104 & 100 \\
\hline
\end{tabular}

Fuente: Padres de familia y líder comunitaria entrevistados, 2010

Sobre las condiciones del hábitat se encontró presencia de aguas negras empozadas (32.7\%); mala disposición de desechos (19.2\%); lotes enmontados $(52.9 \%)$; calles sin pavimentar y en mal estado (100\%), factores que desmejoran la salud y el bienestar de sus habitantes.

Tabla 21. Relaciones de vecindad

\begin{tabular}{|c|c|c|}
\hline TIPO DE RELACIÓN & F & $\%$ \\
\hline Colaboración & 58 & 55,6 \\
\hline Solidaridad & 33 & 31,7 \\
\hline No se relacionan & 13 & 12,7 \\
\hline Total & 104 & 100 \\
\hline
\end{tabular}

Fuente: Las familias que participaron en la investigación, 2010

En cuanto a las relaciones entre vecinos, el $87,3 \%$ mantiene relaciones de colaboración y solidaridad y el $12.7 \%$ no se relacionan.

Tabla 22. Formas de resolver los conflictos y/o problemas comunitarios

\begin{tabular}{|c|c|c|}
\hline SE RESUELVEN & F & $\%$ \\
\hline Individualmente & 46 & 44.5 \\
\hline Colectivamente & 58 & 55.5 \\
\hline Total & 104 & 100 \\
\hline
\end{tabular}

Fuente: Las familias que participaron en la investigación, 2010

En este ítem se encontró que el $55.5 \%$ de las familias generan sinergias para buscar soluciones coniuntas a los problemas comunitarios que les afecta, mientras que el $44.5 \%$ aseguró que se resuelven individual mente. 
Revista de

investigaciones UNAD

Volumen 10. Número 1. Junio 2011

Tabla 23. Actividades compartidas entre los niños y entre adultos

\begin{tabular}{|c|c|c|}
\hline TIPO DE ACTIVIDAD & F & $\%$ \\
\hline Juegos y realización de tareas & 58 & 55,7 \\
\hline Ver televisión & 14 & 13,5 \\
\hline $\begin{array}{c}\text { Jornadas con la comunidad: } \\
\text { limpieza }\end{array}$ & 11 & 10,6 \\
\hline $\begin{array}{c}\text { Fiestas especiales: de cumplea- } \\
\text { nos y de los niños }\end{array}$ & 14 & 6,7 \\
\hline No comparten & 7 & 100 \\
\hline Total & 104 & \\
\hline
\end{tabular}

La tabla 23 registra que el $69.3 \%$ de los niños interactúan entre sí y un 24.1\% de los adul tos comparten actividades de limpieza, fiesta de los niños y cumpleaños. El $6.7 \%$ no se vincula a las actividades programadas en el barrio.

Como parte de la discusión vemos que la cotidianidad y calidad de vida de las familias en estudio está determinada por diversos factores educativos, economicos, habitacionales, ambientales, comunicativos y relacionales, y que las familias resultan afectadas en cuanto a espacios de socializacion y provision de cuidado y bienestar de sus miembros. Sobre este topico se resal ta que el $71.1 \%$ de los miembros adul tos (padres y abuelos) presentan baja preparacion academica, lo cual incide en el tipo de actividad economica que realizan, en los ingresos percibidos y en la forma como satisfacen las necesidades basicas de vivienda, alimentación, educacion y descanso. Sobre este aspecto, Turnbull y Summers (2000) afirman que "las familias y sus integrantes experimentan su maximo nivel de calidad de vida cuando sus, necesidades son adecuadamente satisfechas, sus miembros disfrutan la vida conjuntamente y tienen la oportunidad de perseguir y conseguir metas que son significativas para su vida".

Las viviendas no poseen los espacios y servicios completos;' la pequeña sala sirve al mismo tiempo de comedor; en una o dos habitaciones en promedio, cohabitan cinco seis y mas miembros de la familia, presentandose hacinamiento, fal ta de privacidad y problemas en la convivencia diaria, en detrimento de los vínculos familiares y humanos. A este respecto, estudios realizados por el Banco Interamericano de Desarrollo, el Fondo M onetario Internacional y el Banco mundial (2003) asocian vivienda digna y pobreza, llegando a hablar de pobreza de espacio habitacional, que hace referencia a las.personas que habitan hacinadas en viviendas sobreocupadas, lo que es aplicable a laș familias en el estudio que nos ocupa. A demas, las viviendas en su mayoria carecen de los servicios publicos domiciliarios (76\%), constituyendose en factores detonantes de contaminacion ambiental y de 


\section{BancaPérezContreres}

Cotidianidad familiar y comunitaria con relación al desarrollo humano en familias del barrio La Panela del municipio de

Corozal, Sucre, artículo producto de la investigación

desarrollo humano, puesto que coarta las posibilidades de llevar a cabo las actividades cotidianas, al menos de manera aceptable.

El ambiente de conflicto y desacuerdos en que se desenvuelve casi un $50 \%$ de las familias también es desfavorecedor del desarrollo humano. La tamilia como, instituyente primario, no provee un clima de convivencia y de construcción de identidades personales y sociales equilibradas, lo que impulsa a adoptar comportamientos y conductas que atentan contra el desarrollo integral de los infantes y adolescentes. El poco tiempo compartido en familia es, igualmente, un factor de riesgo para niños, niñas y adol escentes, pues sabemos que la ausencia/presencia de la figura paterna y materna es decisiva en la formación de los hijos y en la construccion de un camino personal que favorezca su desarrollo humano.

En lo comunitario, se encontró que el hábitat no ofrece las condiciones necesarias para la salud física y emocional de la población. Factores contaminantes como aguas negras empozadas, disposicion de desechos y calles sin pavimentar y en mal estado, ponen en riesgo el bienestar fisico de todos los habitantes del barrio; la presencia de lugares sin iluminacion y enmontados genera riesgo de abuso sexual, violaciones y consumo de sustancias psicoactivas.

Por último, el sentido de cooperación y la solidaridad manifiesta al resolver los conflictos a nivel comunitario, es una demostración de civismo y un referente positivo para el desarrollo individual, familiar y grupal. El aunar esfuerzos les ayuda a minimizar los problemas y vicisitudes que afrontan en su cotidianidad.

\section{Conclusiones}

Los adultos mayores consideran que sus experiencias vividas son poco valoradas y aprovechadas en la familia y la comunidad, percepcion que se refuerza cuando el entorno social comienza a estigmatizarlos una vez que sobrepasan los cincuenta años. Este hecho amerita un cambio en ellos mismos, la familia y la sociedad para desarrollar una actitud positiva sobre el lugar que deben ocupar en la cultura y en la sociedad, de manera que puedan mejorar su autoestima, las habilidades comunicativas con los hijos, nietos y miembros de la comunidad y, por ende, su calidad de vida.

El desconocimiento de los padres acerca de la importancia del registro de nacimiento, demanda a nivel local una política 0 estrategia de articulacion de la familia y la oficina del registro civil, que los oriente sobre la necesidad de hacer este tramite, en ejercicio del derecho al nombre y para poder gozar 
Revista de investigaciones UNAD

Volumen 10. Número 1. Junio 2011

de otros beneficios que ofrece el Estado, como la vacunación y la educación, que son indispensables para garantizar el desarrollo pleno de las capacidades fisicas, intelectual es y emocionales.

La detección temprana de comportamientos inadecuados en niños y jovenes prioriza trabajar con la familia la importancia de las normas como dispositivos que orientan el buen comportamiento de los individuos para una mejor convivencia y por tanto una mejor calidad de vida.

Indistintamente de la tipología familiar, nuclear, extensa o monoparental, las familias del barrio La Panela presentan dificultades en su diario vivir para asegurar el bienestar, material y social de sus miembros. Por ello, se insta a las personas que actúan en funcion de intereses particulares a que cambien de actitud, pasando del individualismo a la construccion de una conciencia colectivista que funcione como factor de cohesion en situaciones dificiles, y que favorezca el sentido de pertenencia y el sano desarrollo de la comunidad.

\section{Referencias bibliográficas}

Anguera, M. T. (1998). Tratamiento Cualitativo de datos. En M. T. Anguera, J . Arnau, M. Ato, R. M artínez, J. Pascual y Vallejo, G. M étodos de investigación en psicología. ... Madrid: Síntesis.

Campos, A. (2009). M étodos M ixtos de Investigación: Integración de la investigación cuantitativa y la investigación cualitativa. Bogotá: Magisterio.

Congreso de Colombia, Ley 1098, (2006). Código de la Infancia y Adolescencia. República de Colombia, Ministerio de la Protección Social. Coordinación editorial oficina de comunicaciones y atención al ciudadano. Bogotá.

Consejo Nacional para la superación de la pobreza, (1996). La pobreza en Chile: Un desafío de equidad e integración social. Santiago de Chile: Despertar.

Delgado de Bravo, M. y Failache, S. (1993). El concepto de calidad de vida: Una revisión de su alcance y contenido. Revista Geográfica Venezolana.

Delgado, J . M . (1999). M étodos y Técnicas Cualitativas de investigación en Ciencias sociales. Madrid: Síntesis.

García, N. (2004). Crecimiento Económico y Necesidades Básicas. En Pobreza, Necesidades Básicas y Desarrollo Santiago de Chile: Alfabeta. 


\section{BancapéezContreres}

Cotidianidad familiar y comunitaria con relación al desarrollo humano en familias del barrio La Panela del municipio de Corozal, Sucre, artículo producto de la investigación

Gutiérrez de Pineda, V. y Villa de Pineda, P. (1991). Honor, Familia y Sociedad en la estructura patriarcal. El caso de Santander (2aㅡ ed.). Bogotá: Universidad Nacional de Colombia.

Hannerz, U. (1986). Exploración de la ciudad: Hacia una antropología urbana, Fondo de Cultura Económica, México. En: D. F. Henao. Comunicación y Redes Sociales ( 20).

Kerlinger, F. \& Lee, H. (2002). Investigación del Comportamiento: Métodos de Investigación en Ciencias Sociales. Ciudad de México: Mc Graw Hill.

Maldonado, M. C. (1995). Conflicto, poder y violencia en la familia. En: D. F. Henao..., Comunicación y Redes Sociales. Cali: Universidad del Valle.

Sabeh, E. N. y Verdugo M. A (2000). Revisión crítica del uso del concepto de calidad de vida en la infancia. Manuscrito no publicado, Universidad de Salamanca.

Shoveller, N. (2006). Reglas de integración de los métodos cuantitativos y cualitativos. En A. Campos (Comp.) Métodos mixtos de investigación: Integración de la investigación cuantitativa y la investigación cualitativa. Bogotá: Magisterio.

Turnbully Summers, (2000). En: Verdugo M.A. Calidad de vida en las familias con hijos con discapacidad intelectual, [en línea]. Disponible en: http://www, feaps.org/ biblioteca/ familias y di/capitul05.psf [2011, 20 de septiembre]. 